\title{
Selective Immunoglobulin A Deficiency, Helicobacter pylori Infection, and Strongyloidiasis in a Patient with Adenocarcinoma of the Stomach
}

\author{
Bassel Mazen Dakkak ${ }^{a} \quad$ Yahia Imam $^{b} \quad$ Khalid Ahmed $^{c}$ \\ Ahmed Osman Saleh ${ }^{d}$ Dina Soliman $^{c}$ Meshaal Alanzi ${ }^{a}$ \\ Mohamed A. Yassin ${ }^{c}$ Shehab Mohamed ${ }^{c}$ \\ aDepartment of Internal Medicine, Hamad Medical Corporation, Doha, Qatar; ${ }^{b}$ Department \\ of Neurology, Weill Cornell Medical College, Doha, Qatar; 'Department of Hematology, \\ National Centre for Cancer Care and Research, Hamad Medical Corporation, Doha, Qatar; \\ ${ }^{\mathrm{d} D e p a r t m e n t}$ of Endocrinology, Hamad Medical Corporation, Doha, Qatar
}

\author{
Keywords \\ $\lg \mathrm{A} \cdot$ Stomach $\cdot$ Adenocarcinoma $\cdot$ Strongyloidiasis $\cdot$ Helicobacter pylori
}

\begin{abstract}
Selective immunoglobulin A $(\lg A)$ deficiency is one of many congenital immunodeficiencies. It is associated with several medical condition. It has been shown to be associated with some types of malignancies, some autoimmune disorders, and even with some infections. Here we report a young male with selective IgA deficiency who also tested positive for Helicobacter pylori and strongyloidiasis at the time when he was diagnosed with stomach adenocarcinoma. The presence of IgA deficiency with multiple etiological possibilities such as infections and cancer makes this case unusual.

\section{Introduction}

Although uncommon, selective immunoglobulin A ( $\operatorname{IgA})$ deficiency is the most common primary immunodeficiency. Its pathogenesis mainly involves defects in immunoglobulin class switching, production, and secretion of IgA during maturation of the B cell [1].

Most patients are asymptomatic and are incidentally diagnosed during routine laboratory screening by demonstrating levels below $7 \mathrm{mg} / \mathrm{dL}(0.07 \mathrm{~g} / \mathrm{L})$ in the serum with normal levels of other immunoglobulins when symptomatic, pulmonary infections, autoimmune

Bassel Mazen Dakkak

Department of Internal Medicine, Hamad Medical Corporation Al Rayyan Street

Doha 00000 (Qatar)

bdakkak@hamad.qa
Yahia Imam

Neurology Department, Weill Cornell Medical College

Al Luqta Street, Al Rayyan

Doha 00000 (Qatar)

yzi3001@qatar-med.cornell.edu 
Fig. 1. Esophagogastroduodenoscopy showing fungating gastric mass (see arrows).

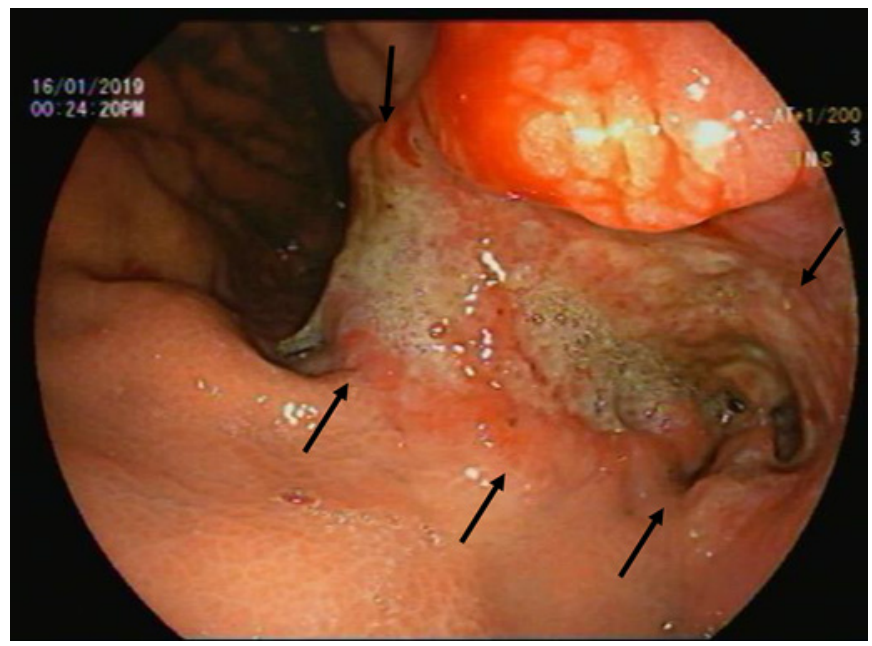

diseases, gastrointestinal disorders, malignancy, and classically transfusion-related reactions are the common presentations [2].

There is no specific treatment; management focuses on addressing concurrent complications and comorbidities [3].

A known association in patients with IgA deficiency is the increased risk of malignancies, particularly gastrointestinal cancers [2].

Here we report multiple associations of IgA deficiency in a single patient. Although those associations have been either reported or hypothesized about in the literature, to our knowledge, this the first report depicting them all together.

\section{Case Presentation}

A 35-year-old Indian male who was previously healthy, presented to the emergency department with epigastric pain for 5 days. This was associated with intermittent episodes of black stool, fatigue, and loss of appetite for 2 weeks prior to presentation and a significant weight loss of $4 \mathrm{~kg}$ in the preceding 6 months. He was found to have microcytic anemia with a hemoglobin of $5.6 \mathrm{~g} / \mathrm{dL}$ and his iron profile was suggestive of iron deficiency anemia. The platelets and the coagulation profile were normal. A blood transfusion was arranged to correct his anemia, but he developed a transfusion reaction on 2 separate occasions with breathing difficulty and restlessness a few minutes after starting the blood transfusion. This was accompanied by hypotension on the second attempt. The blood transfusion was stopped. There was no prior history of blood transfusion or allergy, and since he was young and from an Asian ethnicity, IgA deficiency was suspected. IgA level was sent and came back as low titer $(<0.50 \mathrm{~g} / \mathrm{L})$, with a negative Coombs test. Washed packed red blood cell units were arranged for transfusion. US abdomen showed thickening of the gastric wall measuring up to $1.5 \mathrm{~cm}$ at the pyloric region. CT abdomen with IV contrast showed a circumferential wall thickening of the distal gastric body and pyloric region, with a fungating mass having an exophytic component arising from the posterior aspect of the pylorus with central breakdown suggestive of gastric malignancy.

Gastroscopy was done after 2 days and a circumferential, ulcerating, friable mass that bleeds to touch was found occupying all the antrum, the pylorus, and the distal body of the stomach (shown in Fig. 1). The biopsy from the mass showed invasive gastric adenocar- 
cinoma of the mixed type. Positron emission tomography (PET) scan was done and showed multiple peritoneal, abdominal lymph nodes and bilateral small pulmonary metastases. The patient was subsequently treated with a palliative intent. Other workup showed eosinophilia, and stool microscopy showed Strongyloides species larvae. Stool H. pylori antigen was positive and was confirmed by Warthin-Starry stain on biopsy.

\section{Discussion}

In our case the clinical suspicion started because of repeated transfusion reaction two times within the same day. Therefore, we sent for IgA level. The diagnosis was confirmed and washed RBCS transfused successfully. After labeling our patient with two diagnoses, the stomach cancer and the IgA deficiency, we started to look in the literature for this association and other associations related to it. IgA is the most abundant antibody isotype in the human body and second most abundant immunoglobulin type after IgG in the blood. While its presence in body secretions plays a crucial role in our body's defense against antigens such as bacteria, viruses, and toxins, its role in systemic immunity is still yet to be discovered [1]. The subclass IgA1 is monomeric and is found in the blood circulation, while the subclass IgA2 is dimeric and is found in the mucosal secretions [1]. IgA needs multiple steps to be secreted to its mucosal site of action. First the IgA needs to be produced locally by the plasma cells as a dimer with a J chain attached to make IgA + J complex. This complex then attaches to the Ig receptor located on the basolateral surface of the epithelial cell to stimulate transcytosis, cleavage is done inside the cell into dimeric IgA, then it is secreted to the mucosal lumen.

Although the role of mucosal IgA is well known, the functions of serum IgA is still yet to be discovered. However, receptors for the fragment crystallizable region ( $\mathrm{Fc}$ region) of IgA are present in granulocytes and monocytes and IgA bound to these receptors can initiate phagocytosis. Those receptors may play a role in clearing immune complexes from the serum [4].

Selective IgA deficiency is the most common type of primary immunoglobulin deficiency and is defined as low serum IgA with normal levels of other isotypes. Mucosal IgA levels is not determined. Most patients are asymptomatic as some may have enough secretory IgA to mount a protective immune response. Furthermore, in the majority of cases there is a compensatory increase in the secretory IgM [5, 6]. The incidence and prevalence varies with different ethnic backgrounds, for example, incidence is 1:143 in the Arabian peninsula [7], 1:875 in England and 1:2,600 to 1:5,300 in China [8]. In general, IgA deficiency is more prevalent in Caucasians. In the United States, the frequency is estimated to be 1:223 to 1:1,000 in community studies. These numbers may underestimate the actual incidence and prevalence of the disease because the majority are asymptomatic and there is no current agreement for routine screening [1].

The associations between IgA deficiency and other diseases are well established. It is classically associated with different autoimmune diseases. Additionally, immune-suppressed patients (such as IgA-deficient individuals) have increased risk of certain malignancies. Although the overall cancer risk was not elevated in a study conducted in Sweden and Denmark in patients with IgA deficiency, there was higher frequency of stomach cancer in this group of patients [9]. Moreover, in a study conducted in New York, cancer is more prevalent in patients with IgA deficiency, with gastric malignancy being the most frequent followed by reticuloendothelial system-related malignancy [10]. More evidence pointing to the association between humoral immunity and malignancy is demonstrated by a higher frequency of positivity of IgA in early gastric cancer histology compared with more advanced stages. Such immunohistochemical demonstration suggest that IgA has a role in cancer progression and may influence tumor growth [11].

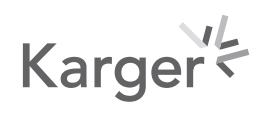


It is postulated that the decrease of IgA seen in IgA deficiency can promote Helicobacter pylori colonization and gastric inflammation, thus promoting carcinogenesis [12]. Although IgA deficiency is associated with gastric cancer, the association between IgA deficiency and H. pylori infection is complex [2]. In animal models, B cell-deficient mice demonstrated near similar colonization rates compared to normal mice, but had higher rates of clearance in the context of severe gastritis [13]. This implies that the production if IgA against $H$. pylori impairs rather than promotes the host defense against $H$. pylori. A postulated mechanism is that IgA binds to H. pylori and helps the bacteria in evading the immune system [14]. However, in theory, the presence of IgA might halt bacterial growth without eradicating the infection. Therefore, IgA deficiency might cause bacterial overgrowth of H. pylori and subsequently increase the risk of gastric cancer. Unfortunately, further studies are needed to assess the question of whether the risk of cancer is greater in seropositive IgA-deficient individuals [2].

There is evidence that selective IgA deficiency is closely related to common variable immunodeficiency (CVID) or even is a different expression of the same disease [9]. A study on gastric pathology in CVID patients confirmed a role for $H$. pylori in gastric carcinogenesis [15].

Another possible association with IgA deficiency is strongyloidiasis. As this infection is usually asymptomatic in immunocompetent individuals, it can be fatal in immunodeficient patients. One of the known risk factors for hyper-infection is hypogammaglobinemia. Cellular immunity appears to be pivotal in preventing the dissemination of Strongyloides, but humoral immunity may be needed to help eradicate the infection as seen with therapeutic failure in patients with hypogammaglobulinemia; therefore, it appears that neither type of immunity alone is sufficient to eradicate the organism [16]. Whereas most patients with strongyloidiasis mount specific IgA responses against larvae antigens that are different from those recognized by IgG, there was no correlation between total serum-specific IgA and the clinical form of the disease in immunocompromised patients. However, the rate of transformation between the rhabditiform larva and the filariform larva (penetrating form) may be regulated by the secretory component of the immune system as IgA specific to both forms was found in most but not all of the patients [17].

Our case is unique because it has all these associations together (IgA deficiency with gastric adenocarcinoma, $H$. pylori and Strongyloides stercoralis infection).

\section{Conclusion}

Selective IgA deficiency is associated with several diseases including malignancies and bacterial and helminthic infections. Physicians should not be deterred from investigating further associations in the right clinical setting. Simple stool test can uncover important information. Also, it is simple, cheap, and available in most healthcare facilities. Unexplained blood transfusion reaction is another clue toward making a diagnosis of IgA deficiency.

\section{Statement of Ethics}

Our research complies with the guidelines for human studies and was conducted ethically in accordance with the World Medical Association Declaration of Helsinki. Our local ethics committee in Hamad Medical Corporation (Medical Research Center) has granted permission for this case report to be published on condition that no patient-identifiable data (including patient name and photograph) are included. The approval was obtained under the following number: MRC-04-20-508. The same manuscript was submitted to the Medical

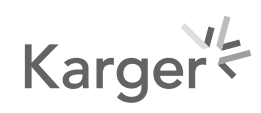


Research Center and was reviewed prior to giving the permission for publication. Informed written consent for publication was not asked for by the Medical Research Center since no data that can potentially and clearly identify the patient was found in the case description.

\section{Conflict of Interest Statement}

The authors declare that they have no conflict of interest with regard to the writing and publication of this article.

\section{Funding Sources}

The authors did not receive funding for this publication.

\section{Author Contributions}

All authors listed on the title page contributed equally.

\section{References}

1 Yel L. Selective IgA deficiency. J Clin Immunol. 2010;30(1):10-6.

2 Ludvigsson JF, Neovius M, Ye W, Hammarström L. IgA deficiency and risk of cancer: a population-based matched cohort study. J Clin Immunol. 2015;35(2):182-8.

3 Vo Ngoc DT, Krist L, van Overveld FJ, Rijkers GT. The long and winding road to IgA deficiency: causes and consequences. Expert Rev Clin Immunol. 2017;13(4):371-82.

4 Cunningham-Rundles C. Physiology of IgA and IgA deficiency. J Clin Immunol. 2001;21(5):303-9.

5 Brandtzaeg P, Karlsson G, Hansson G, Petruson B, Björkander J, Hanson LA. The clinical condition of IgA-deficient patients is related to the proportion of IgD- and IgM-producing cells in their nasal mucosa. Clin Exp Immunol. 1987;67(3):626-36.

6 Klemola T. Immunohistochemical findings in the intestine of IgA-deficient persons: number of intraepithelial T lymphocytes is increased. J Pediatr Gastroenterol Nutr. 1988;7(4):537-43.

7 al-Attas RA, Rahi AH. Primary antibody deficiency in Arabs: first report from eastern Saudi Arabia. J Clin Immunol. 1998;18(5):368-71.

8 Feng L. [Epidemiological study of selective IgA deficiency among 6 nationalities in China]. Zhonghua Yi Xue Za Zhi. 1992;72(2):88-128.

9 Mellemkjaer L, Hammarstrom L, Andersen V, Yuen J, Heilmann C, Barington T, et al. Cancer risk among patients with IgA deficiency or common variable immunodeficiency and their relatives: a combined Danish and Swedish study. Clin Exp Immunol. 2002;130(3):495-500.

10 Cunningham-Rundles C, Pudifin DJ, Armstrong D, Good RA. Selective IgA deficiency and neoplasia. Vox Sang. 1980;38(2):61-7.

11 Takemura K, Hirokawa K, Esaki Y, Mishima Y. Distribution of immunoglobulins and secretory component in gastric cancer of the aged. Cancer. 1990;66(10):2168-73.

12 Vajdic CM, Mao L, van Leeuwen MT, Kirkpatrick P, Grulich AE, Riminton S. Are antibody deficiency disorders associated with a narrower range of cancers than other forms of immunodeficiency? Blood. 2010;116(8): 1228-34.

13 Israel DA, Peek RM Jr. The role of persistence in Helicobacter pylori pathogenesis. Curr Opin Gastroenterol. 2006;22(1):3-7.

14 Akhiani AA, Stensson A, Schön K, Lycke NY. IgA antibodies impair resistance against Helicobacter pylori infection: studies on immune evasion in IL-10-deficient mice. J Immunol. 2005;174(12):8144-53.

15 Zullo A, Romiti A, Rinaldi V, Vecchione A, Tomao S, Aiuti F, et al. Gastric pathology in patients with common variable immunodeficiency. Gut. 1999;45(1):77-81.

16 Segarra-Newnham M. Manifestations, diagnosis, and treatment of Strongyloides stercoralis infection. Ann Pharmacother. 2007;41(12):1992-2001.

17 Genta RM, Frei DF, Linke MJ. Demonstration and partial characterization of parasite-specific immunoglobulin A responses in human strongyloidiasis. J Clin Microbiol. 1987;25(8):1505-10. 